\title{
The study of exposure times and dose-escalation of tick saliva on mouse embryonic stem cell proliferation
}

\author{
Ahmet KOCABAY1,5 (D), Ayyub EBRAHIMI² (D), Ali Cihan TAŞKIN ${ }^{3}$ (D), Sırrı KAR ${ }^{4}$ (D) \\ 1 Translational Medicine Research Center, Koç University, Istanbul, Turkey \\ ${ }^{2}$ Department of Molecular Biology and Genetics, Faculty of Science and Literature, Haliç University, Istanbul, Turkey \\ ${ }^{3}$ Department of Laboratory Animal Science, Aziz Sancar Institute of Experimental Medicine, Istanbul University, Istanbul, \\ Turkey \\ ${ }^{4}$ Department of Biology, Faculty of Arts and Sciences, Namık Kemal University, Tekirdağ, Turkey \\ ${ }^{5}$ Corresponding author: akocabay@ku.edu.tr
}

ASBTRACT: The saliva of ticks contains numerous bioactive molecules with anti-hemostatic and immunomodulatory properties. Due to their abilities of self-renewal and pluripotency, stem cells hold considerable promise in the regenerative medicine and biomedical fields. The present study examines the viability and proliferation of mouse embryonic stem cells (mESCs) following the addition of tick salivary gland extracts obtained from three tick species (Dermacentor marginatus, Rhipicephalus bursa and Hyalomma marginatum) to the mESC medium in different quantities $(0.2,2,20,40,80$, and $160 \mu \mathrm{g} / \mathrm{ml}$ ). On days 2, 5 and 7 of the treatment, the vitality and proliferation of the cells were determined with CellTiter-Glo and morphological tests. The results showed that the culture supplemented with D. marginatus salivary gland extract at a concentration of $80 \mu \mathrm{g} / \mathrm{ml}$ positively affected the proliferation rate of mESC. It was further shown that all concentrations of the salivary gland extracts derived from H. marginatum and R. bursa had a negative effect on the proliferation rate of mESC when compared to the controls.

Keywords: Mouse, stem cell, tick saliva, proliferation.

Zoobank: http://zoobank.org/0F8E7E30-A14D-4E5A-90D9-4335F2636532

\section{INTRODUCTION}

Ticks (Acarina: Ixodida) represented by around 900 species identified to date throughout the world are obligate hematophagous ectoparasites. Unlike many other bloodsucking arthropods, they remain attached to the host skin for several hours to days and need to overcome some difficulties in this feeding process, such as creating a feeding pool in the host skin around their mouth parts, suppressing the blood clotting process, and combating the host immunity (Sonenshine and Roe, 2014) mainly by modulating the innate and acquired immune responses (Kotál et al., 2015; Wikel, 2018). Consequently, saliva of ticks, particularly of ixodid (Ixodidae) species, was reported to be arguably the most complex saliva of any animal (Nuttall, 2019). Up to the present, more than 500 different proteins and peptides have been described in tick saliva (Esteves et al., 2017, Perner et al., 2018). In addition to proteins and peptides, many non-peptidic molecules have been determined, such as nucleoside, adenosine, microRNAs, arachidonic acid derivative, prostaglandins (PGE2, F2 $\alpha$, I2, D2 and A2/B2), and endocannabinoids (Nuttall, 2019). However, molecular composition of saliva can vary considerably depending on the tick species, their biological stages, sex and feeding periods (Kaufman, 2007; Francischetti et al., 2009), and it is known that the molecule diversity and their amounts are particularly elevated in the late/accelerated stage of feeding in females (Leboulle et al., 2002; Mudenda et al., 2014; Karim and Ribeiro, 2015). Some molecules in the saliva of dozens of tick species have been identified to have quite different and effective bioactivity. However, there is no data about the majority of the tick saliva molecules (Mudenda et al., 2014; Ribeiro et al., 2017; Nuttall, 2019). There are a few comprehensive and recent reviews on the composition and role of tick saliva. Tick salivary molecules have a promising therapeutic potential for treatment of some human diseases associated with hemostatic and immunological disorders (Štibrániová et al., 2019; Nuttal 2019; Aounallah et al., 2020). Transcriptome and proteome studies of tick salivary glands (SGs) discovered an enormous protein diversity and unique proteins belonging to novel protein families with unknown functions (Štibrániová et al., 2019). Furthermore, it has been proposed that many substances found in tick saliva can be worth examining for different purposes due to their special properties, such as cytotoxic, apoptotic, antiangiogenetic, immunomodulatory, anti-inflammatory, antiplatelet, and anticoagulant activities (Kazimírová and Štibrániová 2013; Sousa et al., 2015).

Stem cells are undifferentiated cells with the ability of self-renewal and can transform into multiple types of functional and specialized cells under appropriate conditions in the body (in vivo) or in the laboratory (in vitro). These self-renewing cells are derived from the inner cell mass (ICM) of mouse blastocysts (Evans and Koufman, 1981; Martin, 1981). Due to their unique properties, embryonic stem cells (ESCs) have potential for use in a wide range of areas, such as embryology, tissue regeneration, tissue-organ engineering, production of transgenic animals, etc. (Smith and Chepko, 2001; Iijima et al., 2010). 
Although considerable progress has been achieved in stem cell studies since the discovery of ESCs, ESCs culture procedures are still complex and expensive. As such, there is still considerable interest in identifying the culture conditions that enable ECSs to renew themselves preventing their differentiation and promoting their proliferation (Silva-Cote and Cardier, 2011). It is essential that stem cells, which hold great promise in the regenerative medicine and biomedical fields due to their abilities of selfrenewal and pluripotency, be prevented from differentiation, thus preserving their abilities, and it is well known that ESC quality depends on the culture conditions in current practice (Smith and Chepko, 2001; Iijima et al., 2010). In this regard, a great need has been expressed to create culture conditions that mimic a stem cell microenvironment in which the cells can preserve their character and potential (Tamm et al., 2013).

The present study examines the effects of six different concentrations $(0.2 \mu \mathrm{g} / \mathrm{ml}, 2 \mu \mathrm{g} / \mathrm{ml}, 20 \mu \mathrm{g} / \mathrm{ml}, 40 \mu \mathrm{g} / \mathrm{ml}$, $80 \mu \mathrm{g} / \mathrm{ml}, 160 \mu \mathrm{g} / \mathrm{ml}$ ) of salivary gland extracts (SGE) from three tick species (D. marginatus, $R$. bursa and $H$. marginatum) on mouse embryonic stem cells (mESCs) through their addition to a medium containing two inhibitors (PD03259010 and CHIR99021) without serum (Eiselleova et al., 2008). Counts were made and assessed at three different time points (days 2, 5 and 7), at which the experiments were terminated. Viable cell numbers were determined by a CellTiter-Glo® (CTC) test. Morphological characteristics of the mESCs lines obtained at the end of the experiments were examined and analyzed through alkaline phosphatase staining, being a marker of pluripotent cells. This study is intended to improve the efficiency and optimization of traditional stem cell cultivation conditions, and to this end, aims to answer the question of whether tick SGE has an accelerating effect on the proliferation of mESCs..

\section{MATERIALS AND METHODS}

\section{Collection of Salivary Glands from Ticks}

The main material of the study was adult female ticks of the $H$. marginatum, $R$. bursa and D. marginatus species. These ticks originated from Namik Kemal University, Department of Biology, were specific pathogens free and fed on adult New Zealand rabbits (Oryctolagus cuniculus) for continuation. The process of feeding the colonies on rabbits was carried out in the Namı Kemal University Application and Research Center for Experimental Animals, after permission for the study was granted by the Namık Kemal University the Local Ethics Committee for Animal Experiments (07.04.2016 - 9).

For the feeding of the adult ticks, a white cotton fabric bag was placed over the ears of the rabbits, and six unfed adult female and eight male ticks were placed on each ear. The ticks were monitored daily on a regular basis and females observed to have entered the rapid - feeding phase (the last phase of feeding), and mostly engorged female ticks (Sousa et al., 2015) were removed from the ears via forceps with a utmost care so as not to damage the tick, i.e., not to put pressure on its body and not to rupture the mouth parts. The collected female ticks were washed with distilled water and placed in a petri dish containing sterile phosphate buffered saline (PBS) $\left(+4^{\circ} \mathrm{C}\right)$ and their salivary glands were removed with a microsurgery set under a stereo microscope (Patton et al., 2012). The removed salivary glands were separated meticulously from other tissue residues in a separate petri dish in new sterile PBS. The salivary glands were transferred into tubes and washed three times with sterile PBS, and then stored at $-80^{\circ} \mathrm{C}$ until extraction.

\section{Preparation of Tick Salivary Gland Extracts}

The tick salivary glands, were taken from the freezer ($\left.80^{\circ} \mathrm{C}\right)$, disrupted and homogenized with stainless steel beads at $50 \mathrm{~Hz}$. at $+4^{\circ} \mathrm{C}$ for 5 minutes by tissue lyser (until no tissue debris is visible) (Tissue Lyser LT Qiagen). The obtained suspension was transferred into new centrifuge tubes and centrifuged at 15,000 rpm for 5 minutes. Afterwards, the supernatant was removed (salivary gland extract - SGE) and stored at $-80^{\circ} \mathrm{C}$ until needed in the next step (Tietz and Anderson, 1986; Hudson and Hay, 1989).

To prepare the stored tick SGEs for use in the in vitro treatment of the cell culture, they were sterilized by being passed through a $0.22-\mu \mathrm{m}$-filter. A "Thermo Fisher Scientific-Pierce $^{\mathrm{TM}}$ BCA (bicinchoninic acid) Protein Assay" (23225) kit was used to measure the protein concentration and the SGE with determined protein concentrations were divided into stocks with equal concentrations.

\section{Preparation of mESCs}

This study tested at six different concentrations $(0.2,2$, $20,40,80,160 \mu \mathrm{g} / \mathrm{ml}$ ) of SGEs from the different tick species (H. marginatum, $R$. bursa, D. marginatus) in a mouse stem cell (mESC) medium at three different timepoints (early, middle, late stages). As the mESC medium; KnockOut ${ }^{\mathrm{TM}}$ DMEM (Gibco, 10829018) containing 15\% KnockOut Serum Replacement (KOSR, Gibco, 10828028), 1\% non-essential amino acids (NEAA Gibco, 11140050), 1\% penicillin streptomycin (Pen/Strep, Gibco, 15070063), 1\% L -Glutamine (Gibco, 25030081), $0.1 \mathrm{mM}$ 2-Mercaptoethanol (Gibco, 21985-023), $1000 \mathrm{IU} / \mathrm{ml} \mathrm{LIF}$ (Prospec Protein Specialists, CYT-645), 1 MM PD0325901 (Tocris, 4192) and $3 \mu \mathrm{M}$ CHIR99021 (Tocris, 4423) was used (Tamm et al., 2013). The SGEs were then added to the stem cell media at the abovementioned concentrations.

\section{Application of Tick Salivary Gland Extracts to mESCs}

The experiments were designed to run in 96-well culture plates in a petri dish at three timepoints (day 2, day 5 and day 7), with three technical replicates for each group. Before the procedure, the petri dishes were coated with 1\% gelatin for 15-20 minutes, after the gelatin was aspirated, the mitomycin-inactivated feeder cell layer (MEF) was seeded, with equal numbers $\left(\sim 10^{4}\right.$ cells $)$ per well. The MEFs were attached to the gelatin, by being incubated overnight in the D10 medium (DMEM basal medium (Gibco, 41966029), 10\% FBS (fetal bovine serum Gibco, 16000044 ) and a $1 \%$ Pen/Strep medium at $37^{\circ} \mathrm{C}$ and $5 \%$ $\mathrm{CO} 2$ for one day. After overnight incubation, equal num- 
bers of mESCs, were isolated from E13.5 CB6F1 (C57BL/6J X Balb/c) embryos (Taskin et al. 2019) at the Koç University Animal Research Facility / MEFs. D10 was replaced by the ESC medium incubated for one day to allow the mESCs to attach to the base of the culture plates. On the following day (day 0 of the experiment), all of the ESC media in the petri dishes were withdrawn and replaced with a freshly prepared ESC medium containing SGEs at different concentrations. After two days, CellTiter-Glo (CTG) test was performed for early stage experimental groups (day 2). Afterwards, cell viability rate was measured by the CTG test for other experimental groups (day 5 and day 7) (Fig. 3). Every two days following the first day of the experiment, $50 \%$ of the ESC $2 \mathrm{i}$ (PD0325901 and CHIR99021) medium containing SGE was replaced with fresh medium (Czechanski et al., 2014).

\section{Cell Viability Assay}

To examine the effects of the tick SGE on the proliferation of mESCs, the cell viability of each group was analyzed with a CTG test. After the study, results for three different tick species were obtained. CTG test was applied to determine the negative or positive effects of the SGEs on the proliferation of mESCs. The number of viable cells was determined using a "CellTiter-Glo® (CTG) Luminescent Cell Viability Assay" (G7570-Promega) kit, which is an approach that is based on the measurement of the amount of adenosine triphosphate (ATP) in viable cells. During the procedure, 96-well black microplates were used, and $500 \mathrm{MEFs}$ were seeded into each well. Using the petri dishes with the completed experimental process, the Cell Titer-Glo solution was added to the ESC medium at a ratio of 1:10 in accordance with the kit protocol. The culture plates were placed into the reader (Synergy H1, BioTek) and proliferation of the cells in the wells were examined at $567 \mathrm{~nm}$. Relative fold change of cell viability was normalized to untreated negative control (Tamm et al., 2013).

\section{Morphology of mESC Lines}

Mouse stem cell colonies were first selected based on their morphology, such as compact rounded cells and colonies with distinct edges. Pluripotency of the mESC lines were assessed by alkaline phosphatase staining.

\section{Alkaline Phosphatase (ALP) Staining}

ALP staining was performed to demonstrate whether the pluripotent properties of the mESCs exposed to SGEs at different time points had been maintained. After removing the medium, the cells were washed three times with PBS (PBS-Thermo Fisher, 10010-023), after which, 4\% paraformaldehyde was added to the wells which were then incubated for $5 \mathrm{~min}$ at room temperature in a shaking incubator. The cells were fixed with paraformaldehyde and washed twice with PBS. The staining solution was then added, following the protocol (Sigma Cat. 86R$1 \mathrm{KT}$ ), and the cells were then incubated at room temperature for 60 minutes (Czechanski et al., 2014), washed again with PBS and examined under a microscope.
Statistical Analysis

A two-way ANOVA (mixed model) was used for the statistical assessment of the differences between the media containing different tick SGEs and concentrations. The Geisser-Greenhouse correction and Dunnett's test on multiple comparison were used. Differences at $<0.05$ (pvalue) were considered statistically significant. The experiments were carried out in two biologically independent experiments with three technical replicates for each experiment.

\section{RESULTS}

The study made use of the salivary glands of female ticks that were in the last feeding phase, and that had entered the rapid-feeding phase, although the feeding process did not continue in the same way, even in ticks that were fed on the same day. It took 8-12 days for the ticks to reach the desired feeding phase. The total number and weight of the ticks used were as follows: 28 D. marginatus females with an average weight of $0.3167 \mathrm{~g}$ (min $0.2001 \mathrm{~g}-\max$ $0.5558 \mathrm{~g}), 24 \mathrm{R}$. bursa females with an average weight of $0.3355 \mathrm{~g}$ (min $0.2242 \mathrm{~g}-\max 0.5645 \mathrm{~g}$ ) and $26 \mathrm{H}$. marginatum females with an average weight of $0.3712 \mathrm{~g}$ (min $0.2557 \mathrm{~g}-\max 0.6897 \mathrm{~g}$ ). The number of salivary glands obtained from these ticks were deemed sufficient for the study.

The effects of SGE of three tick species (H. marginatum, $R$. bursa and D. marginatus) have been compared on mESC proliferation. For H. marginatum, all the media, with six different concentrations of SGE had a negative effect on the proliferation of mESCs in every stage of the culture (day 2, day 5 and day 7) (Fig. 1). In the cell cultures with the D. marginatus and H. marginatum, the maximum positive effect was observed in the middle stages (day 5) of the cultures with high concentrations of tick SGE (40 $\mu \mathrm{g} / \mathrm{ml}, 80 \mu \mathrm{g} / \mathrm{ml}, 160 \mu \mathrm{g} / \mathrm{ml}$ ) (Figs 1 - 3).

For $R$. bursa, all of the media, with six different concentrations of SGE had negative effects on the proliferation of mESCs in every examined time point of the treatment (day 2, day 5 and day 7) (Fig. 2). In the cell cultures with the SGEs of $H$. marginatum and $R$. bursa separately, the maximum positive effect was observed in the early stages (day 2) of the cultures with low concentrations of the extract $(0.2 \mu \mathrm{g} / \mathrm{ml}, 2 \mu \mathrm{g} / \mathrm{ml}, 20 \mu \mathrm{g} / \mathrm{ml}$ ) (Figs 1 - 2).

For D. marginatus, all the media, with six different concentrations of SGE, had a negative effect on the proliferation of mESCs in the early (day 2) and late (day 7) stages of the culture. Again, the numbers of mESCs in the middle stages (day 5) of the cultures with extracts at a concentration of $40 \mu \mathrm{g} / \mathrm{ml}$ and $160 \mu \mathrm{g} / \mathrm{ml}$ were close to those of the controls. On the other hand, while a positive effect was observed on mESC proliferation in the middle stage (day 5) of the culture medium with an extract at a concentration of $80 \mu \mathrm{g} / \mathrm{ml}$, the medium no longer had a positive effect in the late stage (day 7) (Fig. 3). 


\section{H. marginatum}
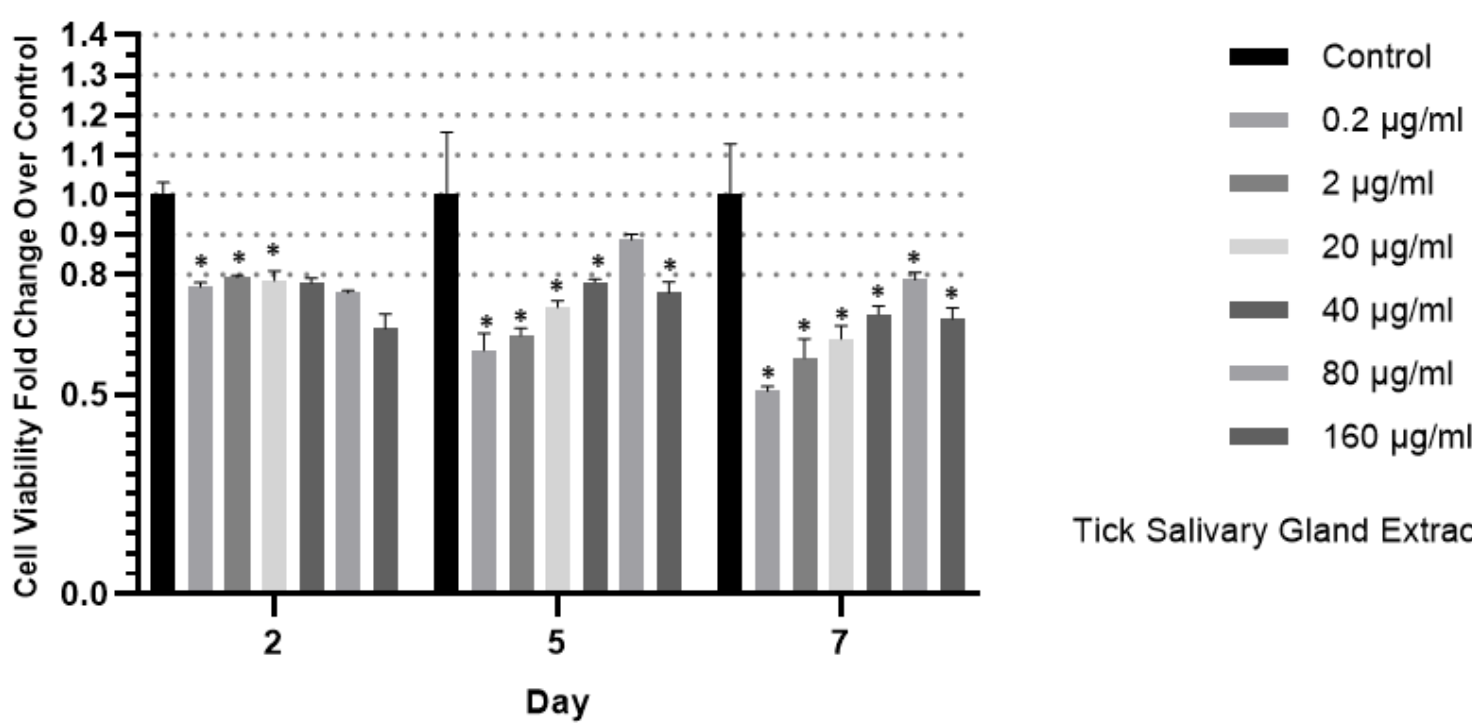

Tick Salivary Gland Extract Concentration

Figure 1. Effect of $H$. marginatum SGE $(0-160 \mu \mathrm{g} / \mathrm{ml})$ on mouse embryonic stem cell proliferation and viability. Values represent relative fold change of cell viability normalized to untreated negative control. The Geisser-Greenhouse correction and Dunnett's test on multiple comparison were used. The results are mean \pm standard deviation (SD) from a representative experiment carried out in triplicate and were seeded in equal amount in 3 different 96 -well cultured plates.
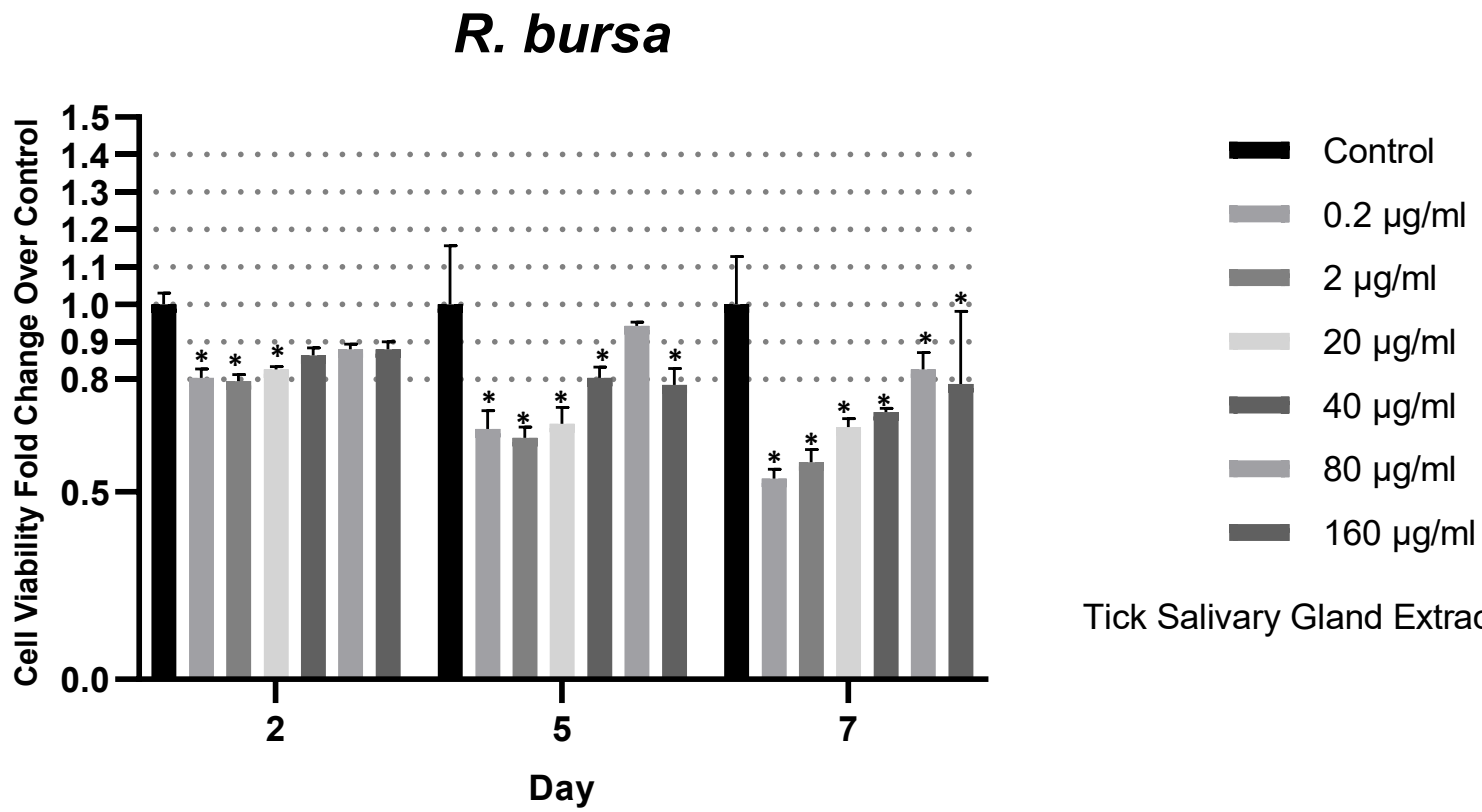

Tick Salivary Gland Extract Concentration

Figure 2. Effect of $R$. bursa SGE $(0-160 \mu \mathrm{g} / \mathrm{ml})$ on mouse embryonic stem cell proliferation and viability. Values represent relative fold change of cell viability normalized to untreated negative control. The Geisser-Greenhouse correction and Dunnett's test on multiple comparison were used. The results are mean \pm standard deviation (SD) from a representative experiment carried out in triplicate and were seeded in equal amount in 3 different 96 -well cultured plates. 


\section{D. marginatus}

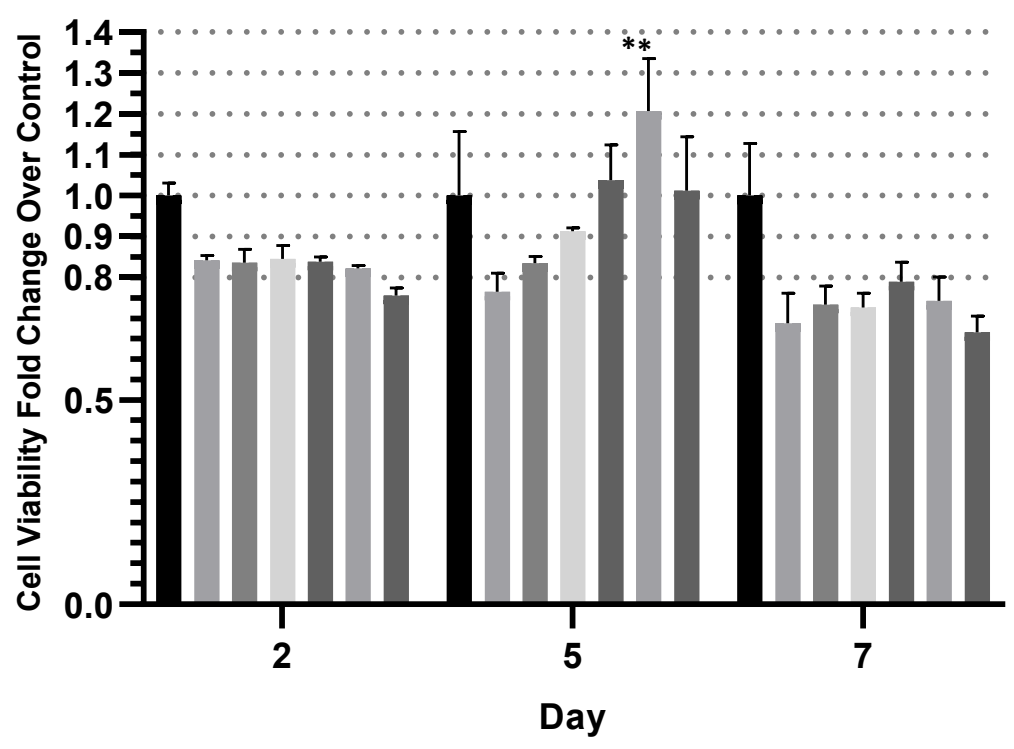

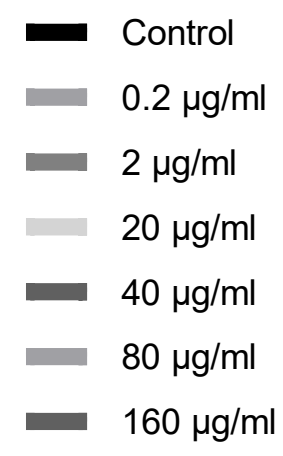

Tick Salivary Gland Extract Concentration

Figure 3. Effect of D. marginatus SGE $(0-160 \mu \mathrm{g} / \mathrm{ml})$ on mouse embryonic stem cell proliferation and viability. Values represent relative fold change of cell viability normalized to untreated negative control. The Geisser-Greenhouse correction and Dunnett's test on multiple comparison were used. All experiments have the $\mathrm{P}$ value $<0.05$, on three different time laps. The results are mean \pm standard deviation (SD) from a representative experiment carried out in triplicate and were seeded in equal amount in 3 different 96 -well cultured plates.

One common remarkable feature noted with all media supplemented by three different tick SGE was that those containing low concentrations of SGE positively the proliferation more positively in the early stages. As the concentrations were increased, the proliferation was higher in the middle stage, however all the values were lower than the control. In these processes, the D. marginatus SGE differed from the others, since the highest proliferation was observed above the control values. This feature was absent for $H$. marginatum and $R$. bursa SGE.

\section{DISCUSSION}

In this study, we investigated the effect of six concentrations of SGE from three tick species on mESC proliferation in the course of three different days. It was demonstrated that specific SGE concentrations SGE of the species increased proliferation of $\mathrm{mESC}$ in middle stages of the culture.

On the other hand, previous studies on tick saliva or SGE reported negative effects on, e.g., endothelial cells. Ornithodoros brasiliensis salivary gland homogenates interfered with endothelial cell proliferation, which is a key phenomenon in wound healing. SGE of $O$. brasiliensis significantly delayed skin wound healing (Reck et al., 2013). This is also supported by previous work showing that saliva of the Ixodes scapularis ( $\leq 1: 500$ dilutions) or salivary gland (0.1-0.5 pairs / assay) dose-dependently inhibited microvascular endothelial cell (MVEC) proliferation (Francischetti et al., 2005). Inhibition was also detected with the saliva of the cattle tick Boophilus microp- lus but not with the salivary gland of Anopheles gambiae (Francischetti et al., 2005). Our results support inhibition effects of tick SGE on mESCs proliferation, except one species. In the future, it will be of interest to investigate the mechanisms behind the positive effects.

Tick salivary glands produce and secrete into the feeding site in the host skin an impressive amount and variety of bioactive molecules modulating hemostatic, inflammatory and immune responses as well as wound healing. The composition of tick saliva is highly complex and changes through the feeding process (Francischetti et al., 2009; Kotal et al., 2015; Blisnick et al., 2017; Šimo et al., 2017; Nuttall, 2019; Wikel, 2018). Studies have reported that tick SGE increases the proliferation on $\mathrm{B}$ and $\mathrm{T}$ lymphocytes and marked degranulation of mast cells and basophils (Gill, 1986; Ushio et al., 1993). The immunomodulatory effect of tick saliva on the production of proinflammatory cytokines released by dendritic cells (DC) stimulated by lipopolysaccharide (LPS) is associated with the inhibition of the activation of extracellular signalregulated kinases (ERK1/2) and mitogen-activated protein (MAP) kinases (Ferreira and Silva, 1998; Oliveira et al., 2010; Lieskovská and Kopecký, 2012). It is known that the inhibition of the ERK1/2 and MAP kinase pathways also positively affects the pluripotency of embryonic stem cells (Ohtsuka et al., 2015).

In recent years several new ES cell culture protocols have been published; however, most laboratories still use the traditional method that involves culturing the ESCs in mitotically inactivated MEFs with media supplemented 
with FBS and LIF (Tamm et al., 2013). In the present study, we added tick salivary gland extracts to the $2 \mathrm{i}$ medium containing KOSR in a standard culture medium and investigated its effects on ESCs.

It is difficult to establish the causality of the mechanism behind the negative effect or the dose-related positive effect specific to D. marginatus SGE observed in this study. It is known that several molecules found in tick saliva interact with many types of receptors which activate different signal transduction pathways involved in different cellular processes such as proliferation, differentiation etc. (Arolas et al., 2005; Brake et al., 2010; Akagi et al., 2012; Carneiro-Lobo et al., 2012; Cao et al., 2013). It is believed that the positive effect specific to $D$. marginatus is attributable to the positive impact of the inhibition of the ERK1/2 and MAP kinase pathways on the pluripotency of the mESCs due to the various proteins in the extract (Oliveira et al., 2010). When the extract concentration was doubled $(160 \mu \mathrm{g} / \mathrm{ml})$, the positive effect on proliferation disappeared, which possibly because the proteins that have a positive effect at low doses negatively affect proliferation in high doses; or some other proteins in SGE that have a negative effect may become more dominant after the dose is increased, although both explanation may be the cause.

In conclusion, it is necessary to diversify the SGE concentrations of $D$. marginatus in the range of $40-160 \mu \mathrm{g} / \mathrm{ml}$. Although maximum proliferation was observed at the 80 $\mu \mathrm{g} / \mathrm{ml}$ concentration in the present study, another concentration may exceed the value we found. Other chemicals or conditions should be tested with the potential to be substituted by the D. marginatus extract in mESC culturing methods. Further studies should also include proteomic analyses to reveal which protein or proteins of the tick SGE affect proliferation. In addition, it is necessary to investigate the pathways affecting the proliferation of mESCs, and the expression levels of the important genes that belong to these pathways.

\section{Authors' contributions}

Ahmet Kocabay: Methodology, investigation, visualization, data curation, formal analysis (supporting), writing original draft. Ayyub Ebrahimi: Visualization, data curation, formal analysis (supporting), writing - original draft. Ali Cihan Taşkın: Project administration, supervision, formal analysis, writing - review \& editing. Sırrı Kar: Conceptualization, project administration, supervision, formal analysis, writing - review \& editing.

\section{Statement of ethics approval}

This study approved by Namık Kemal University the Local Ethics Committee for Animal Experiments (approval number: 07.04.2016-9).

\section{Funding}

This research supported by a grant from TÜBITTAK - The Scientific and Technological Research Council of Turkey (Grant Number: TÜBİTAK-1140638).

\section{Conflict of Interest}

The authors declared that there is no conflict of interest.

\section{Acknowledgements}

The authors gratefully acknowledge use of the services and facilities of the Koç University Research Center for Translational Medicine (KUTTAM), funded by the Republic of Turkey Ministry of Development. The content is solely the responsibility of the authors and does not necessarily represent the official views of the Ministry of Development. This work derived from the first author master thesis.

\section{REFERENCES}

Akagi, E.M., Júnior. P.L., Simons. S.M., Bellini. M.H., Barreto. S.A and Chudzinski-Tavassi, A.M. 2012. Pro-apoptotic effects of Amblyomin-X in murine renal cell carcinoma "in vitro". Biomedicine \& Pharmacotherapy, 66 (1): 6469.

doi: 10.1016/j.biopha.2011.11.015

Aounallah, H., Bensaoud, C., M'ghirbi, Y., Faria, F., Chmelar̆, J. and Kotsyfakis, M. 2020. Tick salivary compounds for targeted immunomodulatory therapy. Frontiers in Immunology, 11: 2440.

doi: 10.3389/fimmu.2020.583845

Arolas, J.L., Lorenzo, J., Rovira, A., Castella, J., Aviles, F.X. and Sommerhoff, C.P. 2005. A carboxypeptidase inhibitor from the tick Rhipicephalus bursa: isolation, cDNA cloning, recombinant expression, and characterization. Journal of Biological Chemistry, 280 (5): 3441-3448. doi: $10.1074 / j b c . m 411086200$

Brake, D.K., Wikel, S.K., Tidwell, J.P. and de León, A.A.P. 2010. Rhipicephalus microplus salivary gland molecules induce differential CD86 expression in murine macrophages. Parasites \& Vectors, 3: 103. doi: 10.1186/1756-3305-3-103

Blisnick, A.A., Foulon, T. and Bonnet, S.I. 2017. Serine protease inhibitors in ticks: an overview of their role in tick biology and tick-borne pathogen transmission. Front Cell Infect Microbiology, 22 (7): 199. doi: 10.3389/fcimb.2017.00199.

Cao, J., Shi, L., Zhou, Y., Gao, X., Zhang, H., Gong, H. and Zhou, J. 2013. Characterization of a new Kunitz-type serine protease inhibitor from the hard tick Rhipicephalus hemaphysaloides. Archives of Insect Biochemistry and Physiology, 84 (2): 104-113. doi: 10.1002/arch.21118

Carneiro-Lobo, T.C., Schaffner, F., Disse, J., Ostergaard, H., Francischetti, I.M., Monteiro, R.Q. and Ruf, W. 2012. The tick-derived inhibitor Ixolaris prevents tissue factor signaling on tumor cells. The Journal of Thrombosis and Haemostasis, 10 (9): 1849-1858. doi: 10.1111/j.1538-7836.2012.04864.x 
Czechanski, A., Byers, C., Greenstein, I., Schrode, N., Donahue, L.R., Hadjantonakis, A.K. and, Reinholdt, L. 2014. Derivation and characterization of mouse embryonic stem cells from permissive and nonpermissive strains. Nature Protocols, 9 (3): 559-574.

doi: 10.1038/nprot.2014.030

Eiselleova, L., Peterkova, I., Neradil, J., Slaninova, I., Hampl, A. and Dvorak, P. 2008. Comparative study of mouse and human feeder cells for human embryonic stem cells. The International Journal of Developmental Biology, 52: 353-363. doi: $10.1387 / \mathrm{ijdb} .0825901 \mathrm{e}$

Esteves, E., Maruyama, S.R., Kawahara, R., Fujita, A., Martins, L.A., Righi, A.A., Costa, F.B., Palmisano, G., Labruna, M.B., Sá-Nunes, A., Ribeiro, R.M.C. and Fogaça, A.C. 2017. Analysis of the salivary gland transcriptome of unfed and partially fed Amblyomma sculptum ticks and descriptive proteome of the saliva. Frontiers in Cellular and Infection Microbiology, 7: 476.

doi: 10.3389/fcimb.2017.00476

Evans, M.J. and Kaufman, M.H. 1981. Establishment in culture of pluripotential cells from mouse embryos. Nature, 292: 154-156.

doi: $10.1038 / 292154 \mathrm{a} 0$

Francischetti, I.M., Mather, T.N. and Riberio, J.M.C. 2005. Tick saliva is potent inhibitor of endothellel cell proliferation and angiogenesis. Thombosis and Heamost, 94 (1): $167-174$.

doi: 10.1160/TH04-09-0566

Francischetti, I.M., Sa-Nunes, A., Mans, B.J., Santos, I.M. and Ribeiro, J.M. 2009. The role of saliva in tick feeding. Frontiers in Bioscience, 14: 2051-2088. doi: $10.2741 / 3363$

Ferreira, B.R. and Silva, J.S. 1998. Saliva of Rhipicephalus sanguineus tick impairs $\mathrm{T}$ cell proliferation and IFNgamma-induced macrophage microbicidal activity. Veterinary Immunology and Immunopathology, 64 : 279-293.

doi: 10.1016/s0165-2427(98)00135-4

Gill, H.S. 1986. Kinetics of mast cell, basophil and eosinophil populations at Hyalomma anatolicum anatolicum feeding sites on cattle and the acquisition of resistance. Parasitology, 93 (2): 305-15. doi: $10.1017 /$ s0031182000051477

Hermant, B., Desroches-Castan, A., Dubessay, M.L., Prandini, M.H., Huber, P. and Vittet, D. 2007. Development of a one-step embryonic stem cell-based assay for the screening of sprouting angiogenesis. BMC Biotechnology, 7: 20.

doi: 10.1186/1472-6750-7-20

Hudson, L. and Hay, F.C. 1989. Practical Immunology. 3rd edition, Blackwell Scientific Publication, Oxford, UK, 47 pp.
Iijima, S., Tanimoto, Y., Mizuno, S., Daitoku, Y., Kunita, S., Sugiyama, F. and Yagami, K. 2010. Effect of different culture conditions on establishment of embryonic stem cells from BALB/cAJ and NZB/BINJ mice. Cellular Reprogramming, 12 (6): 679-688.

doi: $10.1089 /$ cell.2010.0018

Karim, S. and Ribeiro, J.M.C. 2015. An insight into the sialome of the Lone Star tick, Amblyomma americanum, with a glimpse on its time dependent gene expression. PLoS One, 10: e0131292.

doi: 10.1371/journal.pone.0131292

Kaufman, W.R. 2007. Gluttony and sex in female ixodid ticks: how do they compare to other blood-sucking arthropods? Journal of Insect Physiology, 53: 264-273. doi: 10.1016/j.jinsphys.2006.10.004

Kazimírová, M. and Štibrániová, I. 2013. Tick salivary compounds: their role in modulation of host defences and pathogen transmission. Frontiers in Cellular and Infection Microbiology, 3: 43. doi: 10.3389/fcimb.2013.00043

Kotál, J., Langhansová, H., Lieskovská, J., Andersen, J.F., Francischetti, I.M.B., Chavakis, T., Kopecký, J., Pedra, J.H.F., Kotsyfakis, M. and Chmelař, J. 2015. Modulation of host immunity by tick saliva. Journal Proteomics, 128: $58-68$. doi: 10.1016/j.jprot.2015.07.005

Šimo, L., Kazimirova, M., Richardson, J. and Bonnet, S.I. 2017. The essential role of tick salivary glands and saliva in tick feeding and pathogen transmission. Frontiers in Cellular and Infection Microbiology, 7: 281. doi: 10.3389/fcimb.2017.00281

Leboulle, G., Rochez, C., Louahed, J., Ruti, B., Brossard, M., Bollen, A. and Godfroid, E. 2002. Isolation of Ixodes ricinus salivary gland mRNA encoding factors induced during blood feeding. American Journal of Tropical Medicine and Hygiene, 66: 225-233. doi: 10.4269/ajtmh.2002.66.225

Lieskovská, J. and Kopecký, J. 2012. Tick saliva suppresses IFN signalling in dendritic cells upon Borrelia afzelii infection. Parasite Immunology, 34: 32-39. doi: 10.1111/j.1365-3024.2011.01345.x

Martin, G.R. 1981. Isolation of a pluripotent cell line from early mouse embryos cultured in medium conditioned by teratocarcinoma stem cells. Proceedings of the National Academy of Sciences of the United States of America, 78 (12): 7634-7638. doi: $10.1073 /$ pnas.78.12.7634

Mudenda, L., Pierlé, S.A., Turse, J.E., Scoles, G.A., Purvine, S.O., Nicora, C.D., Clauss, T.R.W., Ueti, M.W., Brown, W.C. and Brayton, K.A. 2014. Proteomics informed by transcriptomics identifies novel secreted proteins in Dermacentor andersoni saliva. International Journal of Parasitology, 44: 1029-1037.

doi: 10.1016/j.ijpara.2014.07.003 
Nuttall, P.A. 2019. Wonders of tick saliva. Ticks and TickBorne Diseases, 10: 470-481. doi: 10.1016/j.ttbdis.2018.11.005

Ohtsuka, S., Futatsugi, Y.N. and Niwa, H. 2015. LIF signal in mouse embryonic stem cells. JAK-STAT, 4 (2): e1086520.

doi: $10.1080 / 21623996.2015 .1086520$

Oliveira, C.J., Carvalho, W.A., Garcia, G.R., Gutierrez, F.R., de Miranda, Santos, I.K., Silva, J.S. and Ferreira, B.R. 2010. Tick saliva induces regulatory dendritic cells: MAP-kinases and Toll-like receptor-2 expression as potential targets. Veterinary Parasitology, 167 (2-4): 288-297.

doi: 10.1016/j.vetpar.2009.09.031

Patton, T.G., Dietrich, G., Brandt, K., Dolan, M.C., Piesman, J. and Gilmore, R.D. 2012. Saliva, salivary gland, and hemolymph collection from Ixodes scapularis ticks. Journal of Visualized Experiments, 60: 3894. doi: $10.3791 / 3894$

Perner, J., Kropáčková, S., Kopáček, P. and Ribeiro, J.M.C. 2018. Sialome diversity of ticks revealed by RNAseq of single tick salivary glands. PLOS Neglected Tropical Diseases, 12: e0006410.

doi: 10.1371/journal.pntd.0006410

Reck, J., Marks, F.S., Termignoni, C., Guimaraes, J.A. and Martins, J.R. 2013. Ornithodoros brasiliensis (mouro tick) salivary gland homogenates inhibit in vivo would healing and in vitro endothelial cell proliferation. Parasitology Research, 112 (4): 1749-53. doi: $10.1007 /$ s00436-013-333-3

Ribeiro, J.M.C., Slovák, M. and Francischetti, I.M.B. 2017. An insight into the sialome of Hyalomma excavatum. Ticks and Tick-Borne Diseases, 8: 201-207. doi: 10.1016/j.ttbdis.2016.08.011

Silva-Cote, I. and Cardier, J.E. 2011. Liver sinusoidal endothelial cells support the survival and undifferentiated growth of the CGR8 mouse embryonic stem cell line: Possible role of leukemia inhibitory factor (LIF). Cytokine, 56: 608-615.

doi: 10.1016/j.cyto.2011.08.035

Smith, G.H. and Chepko, G. 2001. Mammary epithelial stem cells. Microscopy Research and Technique, 52: 190-203. doi: $\quad 10.1002 / 1097-0029(20010115) 52: 2<190:: A I D-$ JEMT1005>3.0.CO;2-0

Sonenshine, D.E. and Roe, R.M. 2014. Biology of ticks. Volume 1. Second edition. Oxford University Press, UK, $540 \mathrm{pp}$.

Sousa, A.C.P., Szabó, M.P.J., Oliveira, C.J.F. and Silva, M.J.B. 2015. Exploring the anti-tumoral effects of tick saliva and derived components. Toxicon, 102: 69-73. doi: 10.1016/j.toxicon.2015.06.001

Štibrániová, I., Bartíková, P., Holíková, V. and Kazimírová, M. 2019. Deciphering biological processes at the tickhost interface opens new strategies for treatment of human diseases. Frontiers in Physiology, 10: 830. doi: 10.3389/fphys.2019.00830

Tamm, C., Pijuan, Galitó. S. and Annerén, C. 2013. A comparative study of protocols for mouse embryonic stem cell culturing. PLoS One, 8 (12): e81156.

doi: 10.1371/journal.pone.0081156

Taskin, A.C., Kocabay, A., Ebrahimi, A., Karahuseyinoglu, S., Sahin, G.N., Ozcimen, B., Ruacan, A. and Onder, T.T. 2019. Leptin treatment of in vitro culture embryos increases outgrowth rate of inner cell mass during embryonic stem cell derivation. In Vitro Cellular \& Developmental Biology-Animal, 55: 473-481. doi:10.1007/s11626-019-00367-y

Tietz, N.W. 1986. Textbook of Clinical Chemistry. W.B. Saunders, Philadelphia, USA, 1919 pp.

Ushio, H., Watanable, N., Kiso, Y., Higuchi, S. and Matsuda, H. 1993. Protective immunity and mast cell and eosinophil responses in mice infested with larval Haemaphysalis longicornis tick. Parasite Immunology, 15 (4): 209-14.

doi: 10.1111/j.1365-3024.1993.tb00602.x

Wikel, S.K. 2018. Tick-host-pathogen systems immunobiology: an interactive trio. Frontiers in Biosciences, 23: 265-283.

doi: $10.2741 / 4590$

Edited by: Salih Doğan

Reviewed by: Two anonymous referees

Citation: Kocabay, A., Ebrahimi, A., Taşkın, A.C. and Kar, S. 2022. The study of exposure times and dose-escalation of tick saliva on mouse embryonic stem cell proliferation. Acarological Studies, 4 (1): 1-8. 